\title{
Mutilación genital femenina, entre la cultura y los derechos humanos*
}

[Artículos]

\author{
Jacqueline Blanco Blanco** \\ Omar Antonio Herrán Pinzón ${ }^{* * *}$
}

Fecha de recepción: 4 de octubre de 2020

Fecha de aprobación: 7 de diciembre de 2020

Citar como:

Blanco Blanco, J. y Herrán Pinzón, O. A. (2021). Mutilación genital femenina, entre la cultura y los derechos humanos. Via Inveniendi Et Iudicandi, 16(1).

https://doi.org/10.15332/19090528.6478

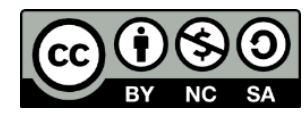

\section{Resumen}

Este artículo analiza la relación cultura-derechos humanos, a partir de la práctica conocida como ablación femenina. Es necesario examinar si en este caso hay correspondencia entre la cultura y los derechos humanos o si, por el contrario, los derechos humanos deben priorizarse para proteger los derechos a la vida y a la sexualidad de las mujeres a quienes se les realiza dicha práctica. Este objeto fue abordado desde los métodos

\footnotetext{
* Este artículo es resultado del proyecto de investigación INV-DER-2556, titulado "Mutilación genital femenina, diversidad cultural y universalidad de los derechos humanos", vinculado al grupo Derecho Público, desarrollado durante el 2018 en la Universidad Militar Nueva Granada (Bogotá, Colombia).

** Doctora en Sociología Jurídica e Instituciones Políticas, magíster en Historia, especialista en Filosofía del Derecho y Teoría Jurídica, y Licenciada en Ciencias Sociales. Docente e investigadora de la Facultad de Derecho de la Universidad Militar Nueva Granada (Bogotá, Colombia). Correo electrónico: jacqueline.blanco@unimilitar.edu.co; ORCID: https://orcid.org/0000-0001-7148-4856

*** Magíster en Derecho Procesal Penal y abogado. Docente e investigador de la Facultad de Derecho de la Universidad Militar Nueva Granada (Bogotá, Colombia). Correo electrónico: omar.herran@unimilitar.edu.co; ORCID: https://orcid.org/00000001-7731-0996
} 
dialéctico y lógico, pues permiten el estudio teórico y conceptual para comprender la ablación desde lo cultural y desde lo jurídico. El análisis concluye que la ablación lesiona la vida y la intimidad de las mujeres; por lo tanto, de tratarse de una manifestación cultural, esta es contraria a los derechos de las mujeres que son sometidas de manera inconsulta.

Palabras clave: ablación, mujeres, cultura, derechos humanos.

\section{Female genital mutilation, between culture and human rights}

\section{Abstract}

This article analyzes the relationship between culture and human rights, based on the practice known as female circumcision. It is necessary to examine whether in this case there is a correspondence between culture and human rights or whether, on the contrary, human rights should be prioritized to protect the rights to life and sexuality of women who undergo this practice. This topic was approached from the dialectical and logical methods, since they allow the theoretical and conceptual study to understand female circumcision from a cultural and legal perspective. The analysis concludes that female circumcision harms the life and intimacy of women; therefore, being a cultural expression, it is contrary to the rights of women who are subjected to it in an unconsulted manner.

Keywords: female circumcision, women, culture, human rights.

\section{Introducción}

En marzo del 2007, los diarios colombianos hicieron pública una noticia acerca de una práctica que hasta la fecha había sido desconocida en el territorio nacional: se trataba de la ablación o mutilación genital aplicada a las niñas indígenas pertenecientes a la etnia embera chamí, asentada en el municipio de Pueblo Rico, Risaralda, en el Eje Cafetero. Según el diario, tres niñas de la comunidad, una de 17 días de nacida y las otras dos de 16 
días, habían sido sometidas a esta ancestral práctica por parte de las parteras del grupo, con el conocimiento de los mayores y la aquiescencia de los padres (Salazar, 2010).

La alarmante noticia llegó a oídos de la Defensoría del Pueblo y otros organismos nacionales e internaciones, quienes de inmediato propusieron una serie de programas que pudieran ser aceptados por los miembros de la comunidad, con el objeto de poner fin a la práctica de la ablación. Las reflexiones en torno al tema se extendieron hasta diciembre del 2010, cuando los embera chamí anunciaron el fin de la práctica.

A diferencia de lo ajeno que pudiera resultar la temática para los colombianos, el número de mujeres sometidas a la ablación en todo el mundo es tristemente grande: 200 millones es la cifra aproximada que maneja la Organización Mundial de la Salud (2020); sin embargo, los informes más recientes advierten que la mutilación genital femenina ha disminuido gracias a la expedición de leyes que, además de prohibir la práctica, establecen sanciones a los padres y a los médicos que intervengan en el proceso. El caso más reciente de legislación expedida para prohibir la denominada circuncisión tipo III ocurrió en Sudán, en abril del 2020 (véase Walsh, 2020).

La mayor dificultad frente a esta práctica está en lo que Laura Nuño, citada por Ludeña Silverio (2018), ha descrito así: "cuando las normas culturales se enfrentan a las normas legales, suele prevalecer la primera" (s. p.). Esta afirmación plantea la necesidad de romper con algunas tradiciones que son amenazas para la pervivencia del grupo que las practica. Entonces, se trata de una forma de "desculturización", en palabras de Ludeña Silverio: de determinadas experiencias que causan subvaloración del género femenino a partir de formas tradicionales respaldadas por la religión y fincadas en la predominancia de sociedades patriarcales para las que el hombre, varón del grupo, es su miembro más preciado. 
La pregunta que orientó el desarrollo de este artículo es: ¿̇la ablación femenina es una práctica cultural que define la ancestralidad en el interior de una comunidad o se trata de una amenaza a la pervivencia de las culturas, por cuanto afecta los derechos fundamentales (a la vida y a la sexualidad, específicamente) de las mujeres a quienes se les practica? Por su parte, el objetivo propuesto señala que es preciso identificar los límites de la práctica de la ablación en cuanto a lo cultural y lo jurídico (Cortés, 2016). En lo cultural, es necesario en nombre de la solidaridad del grupo porque amenaza la vida de las mujeres, multiplicadoras de cultura; en lo jurídico, para evitar la vulneración de los derechos fundamentales de las mujeres, específicamente los relacionados con la vida y la sexualidad (Kymlicka, 1996, p. 8).

\section{Marco teórico}

El abordaje de la temática se hizo mediante el análisis de los conceptos cultura e identidad cultural, por considerarlos esenciales en la comprensión de la problemática, al tiempo que demanda el planteamiento de un debate epistemológico que permita un punto intermedio entre la comprensión de la tradición-ancestralidad y la perspectiva occidental fundada en los derechos humanos. El soporte teórico de la discusión se encuentra en las teorías de Will Kymlicka (1996) sobre el multiculturalismo; también, en algunos aportes de Charles Taylor, en lo que respecta a la pluriculturalidad, así como de Pierre Bourdieu en cuanto al campo cultural, y de Thomas Kuhn con su teoría de la inconmensurabilidad, además de otros autores que abordan con claridad el concepto de cultura e identidad cultural (Rodríguez, 2016). 


\section{Métodos}

Los métodos utilizados fueron la dialéctica y la lógica jurídica, porque posibilitan la comprensión de la norma a partir del contexto y dan lugar a la definición de diversos conceptos, obtenidos gracias a la interacción con diversas concepciones teóricas.

El artículo se desarrolla en dos partes: 1) el debate entre los derechos culturales y los derechos fundamentales, y 2) la postura de la Corte Constitucional colombiana frente a los derechos culturales. En consecuencia, el debate teórico entre cultura y derechos humanos, sugerido en el título y ampliado en la primera parte, se particulariza en la segunda parte del documento al intentar acercarse a lo ocurrido en el interior de la comunidad embera chamí, un caso realmente novedoso en materia de sociología jurídica.

\section{Resultados}

\section{El debate entre los derechos culturales y los derechos fundamentales}

En la tarea por comprender a la sociedad en su particular forma de ser, pensar, sentir y manifestar es imprescindible adoptar una posición dialógica que lleve al entendimiento entre las dos partes: la primera ha hecho suya toda forma de comportamiento y ha normalizado los acontecimientos que en la comunidad no solo resultan cuotidianos, sino que además hacen parte de su visión de mundo; la segunda, generalmente, muestra su desconcierto frente a lo que le es completamente ajeno a su entorno (Ortega et ál., 2020). El problema está en que, para la segunda parte, las prácticas de la primera son calificadas en grado sumo de absurdas, razón por la cual deben ser erradicadas del grupo social observado y, al amparo del amplísimo concepto de los derechos humanos, 
se desconoce que la tradición ha convertido en normal lo que para otros puede resultar extraño o tal vez inadmisible (Chaparro, 2020).

Cuando organizaciones internaciones como la National Geographic revelan algunos acontecimientos que a diario ocurren en los rincones más apartados del mundo, se aprecia, recurriendo a Thomas Kuhn en $L a$ estructura de las revoluciones científicas (2007), lo inconmensurable que resulta conocer, entender y atender las más variadas realidades a las cuales se aferran pequeños grupos humanos. Dichas realidades hacen parte de su naturaleza social, sin que sobre ellas medie precepto jurídico o normativo de carácter externo.

Así, por ejemplo, se tiene conocimiento de la existencia de las denominadas “mujeres kayan", en Tailandia, quienes utilizan una serie de gruesos collares para estirarse el cuello con el objeto de causar un impacto favorable a los ojos de los hombres de su etnia. El número de anillos que componen el collar dan cuenta de la edad y la riqueza de quien lo porta. Estos objetos no podrán ser retirados por ninguna razón y en ningún momento de la vida (Beech, 2020).

En esta misma línea se encuentra el denominado "pie de loto", un proceso aplicado a las niñas en la China que llegó a su final a comienzos del siglo $\mathrm{XX}$, y que consistía en vendar los pies hasta lograr llevar los dedos a la planta del pie, lo que causaba dolores extremos. Las niñas a los siete años de edad empezaban a recibir este tratamiento por parte de su madre. Según las investigaciones de Laurel Bossen y Hill Gates en su libro Bound Feet, Young Hands (2017), lo hacían para asegurar un buen matrimonio, dado que era del agrado de los hombres encontrar a una mujer dedicada a los trabajos manuales, sin otra posibilidad que la de estar en casa produciendo para la economía del hogar a través de actividades como hilar. Esto se lograba gracias a la limitación del movimiento a causa de la 
deformación de los pies, por los vendajes a los que habían sido sometidos. Por esto, el libro lleva por título Pies vendados, manos jóvenes.

El origen de tan disímiles comportamientos debe apreciarse en la existencia misma de diversos grupos sociales con particulares conceptos morales y formas de ver y entender el mundo, lo cual lleva al establecimiento de códigos y pretensiones absolutamente singulares (Ortiz Quiroga, 2013). Con esta consideración se entiende, entonces, que los preceptos sociales son el resultado de una realidad dinámica que plantea la necesidad de democratizar los tratamientos jurídicos alrededor de los cuales se dé primacía a las diferencias, por cuanto se descarta la intención del estatismo u homogenización social, tan favorables a la discriminación y al apartheid (González Cortés, 2020).

Acerca del tema, la profesora Margarita Ramírez afirma que "la identidad cultural que comparte un grupo o una población implica un sentido personal de lealtad” (Ramírez y Mazariegos, 1993, p. 40). Por su parte, Alfredo López-Austin (1989) considera que la "identidad cultural son los elementos en los cuales basa un grupo su diferencia" (p. 20). Para Richard Wilson (1993), la "identidad cultural se concibe como un sentido de pertenencia, que tienen los miembros de un pueblo en relación con los elementos propios del grupo, definiendo estos como sagrados” (p. 34). Para Santiago Bastos y Roddy Brett (2010), la identidad "no solo se basa en el deseo de formar parte de un grupo, sino en el sentido de pertenencia que se tenga de los elementos que determinan la identidad cultural de este grupo, así como el desarrollo de los usos y costumbres de la cultura" (p. 20), algo así como la máxima atribuida a Julio César, según la cual no solo hay que ser, sino también parecer. Esto determina la necesidad de pertenecer a la etnia y comportarse de acuerdo con sus normas.

Así, pues, la identidad cultural puede definirse a partir de elementos comunes como: 1) la existencia de tipologías o rasgos específicos que se 
comparten en el interior de un grupo determinado; 2) la lealtad hacia dichos rasgos que, sumados e integrados a su visión de mundo, constituyen los fundamentos sagrados por los cuales se rigen, y 3) la participación efectiva como miembro de la comunidad, en cuanto a hacer parte de las prácticas establecidas por el grupo. Hasta aquí se supone que la práctica de la ablación es un procedimiento que responde a unos códigos sociales dirigidos a las mujeres de la comunidad, que guardan los preceptos establecidos por un patrón masculino impuesto. Sin embargo, para entenderlo como un tema identitario, habría que verlo desde un espacio eminentemente interno, más desde la intimidad femenina, contradiciendo todo reconocimiento social externo, es decir, lejos de constituir un elemento cultural prevalente a los ojos de la sociedad mayoritaria.

Ahora bien, si la ablación no logra ser un elemento manifiesto, público y predominante de la identidad cultural en una comunidad ancestral, pese a ser una práctica generalizada y compartida, ¿constituye, entonces, una manifestación de su cultura? Procedamos a examinar lo que es cultura. Ricardo Lima Soto (1995) ha sostenido que la cultura es "una práctica social con arraigo entre la mayor parte de los integrantes de una comunidad” (p. 95), que se transmite por generaciones. En la versión de Antonio Gallo (1998):

[...] la costumbre es el medio de cohesión que implica un modo de vivir, un modo de vestir, un modo de alimentarse, un modo de comunicarse, etc., que forman una textura, un tejido con el cual el hombre, dentro del grupo, llega a ser hombre libre. (p. 13)

Por su parte, Dagoberto Vásquez Castañeda (1983) menciona que "la costumbre se encuentra inmiscuida dentro de lo que las tradiciones definen como prácticas de actos con un significado" (p. 9). Rafaela Macías 
Reyes (2011) sostiene que las "tradiciones son costumbres" (p. 62) y, de esta manera, se abre la posibilidad de revisar si toda práctica es verdaderamente histórica al interior de la comunidad y, en algunos casos, originaria; esto es, si tanto los usos sociales como la cosmovisión y las normas se han desarrollado en el interior del grupo desde tiempos remotos y si las generaciones las han conservado, modificadas o no, como parte de su esencia. Estas consideraciones nos remiten a tres aspectos: 1) la cultura traduce una práctica social generalizada, es decir, compartida por la etnia en este caso; 2) el hecho de que la práctica se repita por generaciones, y 3) que tenga un significado. Sin embargo, la ablación es una experiencia improbada y desconocida por sus víctimas, que responde a una posición egoísta del género masculino.

Ahora bien, la cultura elevada al rango de derecho humano fundamental demanda la protección de los actos de la vida diaria de una sociedad que los ha hecho suyos, los ha normalizado y los ha heredado por generaciones. En este caso, ¿cómo actuar jurídicamente frente a la ablación? Según Véronique Champeil-Desplats:

[...] los derechos culturales tienden a descuidar la individualidad para erigir en sujeto de derecho a grupos o colectividades (minorías, comunidades, pueblos) más o menos circunscritos por criterios particularistas e identitarios: prácticas ritualísticas o religiosas, lenguas, códigos sociales... Cualquier negación de los derechos que identifican culturalmente el grupo constituye entonces un etnocidio. (p. 105)

Si bien la autora advierte que atentar contra los derechos que definen la identidad de un grupo es atentar contra su supervivencia cultural, pese a la afirmación de que la ablación no es una clara manifestación de la cultura de un pueblo, la suspensión de esta acción no afecta su valor histórico y cultural. Para comprender mejor el tema, la autora enfrenta los derechos culturales con los fundamentales, y menciona el dilema que se presenta 
con la mutilación genital femenina, donde "preservar la identidad del grupo se hace a expensas de los derechos fundamentales del individuo. Por el contrario, preservar la vida del individuo lleva a rechazar las pretensiones a la fundamentalidad de las prácticas identitarias del grupo" (p. 105).

La posición expuesta por la profesora Champeil-Desplats respalda la afirmación acerca de que la ablación, como práctica, encaja en las categorías que describen tanto a la identidad cultural como a la cultura. Sin embargo, es una verdadera amenaza para los derechos fundamentales de las mujeres que ven amenazada su capacidad sexual y reproductiva. Con todo, la Constitución Política de Colombia de 1991, en sus artículos 7 y 8 , ha declarado el reconocimiento y la protección a la diversidad cultural, por considerarlas parte de la riqueza nacional. Lo que no es fácil establecer, no solo en la Constitución colombiana, sino también en todas las que han optado por incluir los derechos culturales, son las variaciones que la cultura implica, pues esto determina que sea la sociedad mayoritaria la que establezca la normalización de las manifestaciones culturales de los diferentes pueblos y termine definiendo las posibilidades de reconocimiento constitucional que ellas tengan. Al final, y ante lo impredecibles que pueden resultar las manifestaciones culturales, se ha optado por resolver el asunto una vez se presente, sin posibilidad de advertir políticas al respecto.

No obstante, la Corte Constitucional ha concedido protección a la cultura con la igual amplitud constitucional: "la cultura fue reconocida en la Constitución de 1991 como un pilar fundamental que requiere especial protección, fomento y divulgación del Estado" (C. C., Sentencia C-742/o6). Por su parte, la Declaración de Naciones Unidas de los Derechos de los Pueblos Indígenas reconoció, de la misma forma, que estos pueblos tienen 
derecho a: "practicar y revitalizar sus tradiciones y costumbres culturales" (C. C., Sentencia T-001/12).

La protección generalizada a la cultura de los pueblos, así como la autorización expresa y abierta a "revitalizar sus tradiciones y costumbres", remite a un nuevo interrogante relacionado, en esta ocasión, con la originalidad del grupo humano. Sin duda, acontecimientos como la colonización europea o la indetenible colonización cultural moderna complican la posibilidad de encontrar una cultura realmente original. De acuerdo con el profesor Hugues Cayzac (2001):

[...] toda cultura es básicamente pluricultural. Es decir, se ha ido formando, y se sigue formando, a partir de los contactos entre distintas comunidades de vidas que aportan sus modos de pensar, sentir y actuar [...] es a partir de estos contactos que se produce el mestizaje cultural, la hibridación cultural. (p. 16)

De vuelta a la noticia mencionada en la introducción de este artículo (Salazar, 2010), en lo concerniente al rechazo manifestado por la sociedad mayoritaria colombiana a los casos de ablación femenina en los emberachamí, se pone de relieve el efecto pluricultural al que están sometidos los pueblos indígenas en Colombia, lo cual se debe a dos razones: por un lado, a la correspondencia que debe guardarse con la Constitución de 1991, que en su artículo 246 señala que "las autoridades de los pueblos indígenas podrán ejercer funciones jurisdiccionales dentro de su ámbito territorial, de conformidad con sus propias normas y procedimientos, siempre que no sean contrarias a la Constitución y a leyes de la República”. Por otro lado, la cohabitación de diferentes culturas en un mismo espacio geográfico provoca tensiones acerca del comportamiento social de acuerdo con los códigos mayoritarios: 
[...] la sociedad de acogida suele ser hegemónica y suele establecer jerarquías legales y sociales que colocan a los otros grupos en inferioridad de condiciones, lo que lleva al conflicto, al menosprecio, a la creación de estereotipos y prejuicios dificultando la convivencia social, siempre en detrimento de los grupos más débiles. (Bastos y Cumes, 2007, p. 15)

La pluriculturalidad es el resultado de las enormes e irreconciliables distancias culturales construidas durante siglos por las sociedades. A ese mismo nivel de diversidad se encuentran los valores que cada cultura ha definido para sí: "podría decirse que representan diferentes maneras de ser humano. Pero al final, quizá no exista una manera de arbitrar entre ellas cuando chocan entre sí. Quizá sean simplemente inconmensurables” (Taylor, 1996, p. 77).

Retomando el concepto de pluriculturalidad y su implicación, debe decirse que la influencia de la sociedad mayoritaria, en este caso la occidental, sí contribuye a determinar y a modificar en gran medida lo que es conveniente para las sociedades más pequeñas, numéricamente, lo cual las convierte en fragmentos de culturas por años influenciadas por variadas conductas. Lo que necesita definirse aquí es si conviene o no mantenerse fiel a esta historia: "Ya no se puede seguir afirmando que todas las personas necesitan arraigarse en la cultura concreta, en la que ellas y sus ancestros se criaron” (Waldron, 1992, p. 762) (traducción propia).

La globalización y el mestizaje abren la puerta a un desarraigo creativo, enriquecedor, liberador. El protagonista de nuestra época no es el campesino recluido en la cultura ancestral, sino, por ejemplo, el estudiante francés que escucha rai argelino en un aparato japonés de $h i-$ $f i$, se alimenta de pasta italiana y fast food norteamericana, chapurrea español con los inmigrantes sudamericanos que viven su barrio, lee a novelistas rusos, practica meditación budista y artes marciales coreanas, 
y abarca cibernéticamente el orbe a través de Internet. (Contreras Peláez, 2001, p. 151)

La consideración del profesor Contreras Peláez está relacionada con la posibilidad de romper el gueto que puede surgir de estos círculos sociales, pero deja claro que es importante pertenecer a una cultura determinada; por ello, insiste en la necesidad de conservar los valores más originales de la cultura, siempre que no niegue los beneficios que la modernidad ha proporcionado al mundo moderno.

En cuanto al impacto que ejerce lo externo sobre lo interno, o lo mayoritario sobre lo minoritario, Pierre Bourdieu especifica algunos aspectos externos que intervienen, como lo político, lo económico, lo profesional y lo mediático, para luego analizar sus efectos en la cultura, afirmando que su actuar casi imperceptible los convierte en peligrosos detonantes (Moraña, 2014). Luego, desde su teoría de los campos, observa cómo estos se convierten en un espacio de lucha entre los que pretenden redistribuir el poder (Moreno Pestaña y Vázquez García, 2006, p. 223), de donde se origina la relación dominante-dominados que lleva a la existencia de culturas de exclusión que, en América Latina, ha encontrado particular recepción entre las culturas indígenas y campesinas (Giménez Montiel, 2016).

\section{La Corte Constitucional colombiana frente a los derechos culturales}

La Constitución Política de Colombia menciona en 28 oportunidades la palabra cultura para indicar el nivel de compromiso que el Estado adoptó desde 1991 con la tolerancia, el reconocimiento y la protección de las diferencias entre los grupos humanos que habitan el territorio, cuyo fundamento constitucional se halla en los principios de dignidad humana, respeto a la diversidad y protección de las minorías (C. C., Sentencia T605/92). El reconocimiento a las diferencias responde a "la aceptación de 
la alteridad ligada a la aceptación de la multiplicidad de formas de vida y sistemas de comprensión del mundo diferentes de los de la cultura occidental" (C. C., Sentencia T-380/93). Visto desde otro ángulo, se trata de la superación de la homogeneización, en principio excluyente y luego asimilacionista, a la que fue sometida la nación colombiana por más de tres siglos.

La Corte Constitucional ha sentado importantes posiciones que permiten entender algunas prácticas de los grupos indígenas que reclaman la validación de sus rasgos culturales originales, de cara a lo que la Constitución ha denominado "límites" de la autonomía en la Jurisdicción Especial Indígena (JEI) y que, a consideración de la sociedad mayoritaria u occidental, rozan con formas de violación a los derechos humanos.

En el marco del reconocimiento que el Estado hizo de las particularidades de los individuos, la Sentencia T-523/97 analiza el significado de la flagelación como sanción legítima entre la etnia paez o nasa, y manifiesta lo siguiente:

El fuete consiste en la flagelación con "perrero de arriar ganado", que en este caso se ejecuta en la parte inferior de la pierna. Este castigo que se considera de menor entidad que el cepo, es una de las sanciones que más utilizan los paeces. Aunque indudablemente produce aflicción, su finalidad no es causar un sufrimiento excesivo, sino representar el elemento que servirá para purificar al individuo, el rayo. Es, pues, una figura simbólica o, en otras palabras, un ritual que utiliza la comunidad para sancionar al individuo y devolver la armonía. En este caso, y al margen de su significado simbólico, la Corte estima que el sufrimiento que esta pena podría causar al actor no reviste los niveles de gravedad requeridos para que pueda considerarse como tortura, pues el daño corporal que produce es mínimo. Tampoco podría considerarse como una pena degradante que "humille al individuo groseramente delante de otro o en su mismo fuero interno", porque de acuerdo con los elementos 
del caso, esta es una práctica que se utiliza normalmente entre los paeces y cuyo fin no es exponer al individuo al "escarmiento" público, sino buscar que recupere su lugar en la comunidad. (C. C., Sentencia 523/97)

En la misma línea, donde fueron analizados jurídicamente los efectos y la legitimidad del fuete como forma de castigo entre los paeces, está el uso del cepo entre los embera chamí. Al final, la Corte Constitucional (2012) se refirió a ambos métodos por igual: "[...] la imposición [d]el fuete junto con otra sanción de mayor entidad son aceptables dentro de la justicia indígena” (C. C., Sentencia T-001/12).

Pero, sin duda, un acontecimiento que causó revuelo entre los colombianos no indígenas fue el resuelto en la Sentencia T-o3o de 2000. Se trataba de un nacimiento de gemelos en la etnia u'wa, denominado por ellos nacimiento múltiple. La razón del proceso judicial se debió a que los menores fueron entregados al Instituto Colombiano de Bienestar Familiar (ICBF), debido a que la comunidad rechazaba el nacimiento de varios niños en un mismo parto por considerarlo social y religiosamente contaminante. En esta ocasión, la Corte consideró que los derechos de los niños estaban por encima de la identidad cultural del pueblo u'wa y ordenó

[...] constituir un grupo interdisciplinario para que, bajo la coordinación de la Dirección Seccional del ICBF, Agencia Arauca, señalara el momento oportuno del traslado de los menores, recomendara los tratamientos a seguir, e ilustrara a la familia y a la comunidad U'wa sobre los cuidados de los menores, una vez estos retornasen a su comunidad. (C. C., Sentencia T-030/oo)

Igualmente, se indicó el seguimiento del proceso, a partir del cual tenían que rendir informes periódicos a la Sala de Decisión Civil-Familia del Tribunal Superior de Cúcuta. 
Otro caso de singular importancia lo desarrolla la Sentencia C-882/11, al afirmar que:

El uso, consumo y cultivo de la hoja de coca en las comunidades indígenas hace parte de sus costumbres ancestrales, es decir, es una práctica protegida por los derechos a la identidad cultural y a la autonomía de los pueblos indígenas y, por tanto, amparada por el principio de respeto y protección de la diversidad étnica y cultural. (C. C., Sentencia C-882/11)

Ahora bien, tratándose de los alcances que puede tener el derecho a la identidad en Colombia, la Corte fue explícita al mencionar la despenalización de la coca, bajo el argumento de que restringir la identidad cultural a un espacio geográfico determinado pone en riesgo la cultura y traduce políticas de segregación (C. C., Sentencia C-882/11). Igualmente, la Corte ha reconocido que:

[...] la hoja de coca es un elemento fundamental desde el punto de vista cultural, religioso, medicinal y alimenticio, entre otros, para varias comunidades indígenas del país; por esta razón, algunos instrumentos normativos y la jurisprudencia constitucional han reconocido que los usos ancestrales de esta planta se encuentran amparados por la Constitución, en particular, por el derecho a la identidad cultural y autonomía de dichas comunidades. (C. C., Sentencia C-882/11)

El tratamiento que la Corte Constitucional ha reconocido y autorizado para el cultivo y consumo de coca en el interior de las comunidades indígenas colombianas excluye el manejo como alucinógeno desde el mundo occidental; sin embargo, la Corte es enfática en indicar la protección de la práctica por tratarse de una acción eminentemente original de estos pueblos americanos, no trasplantada por los colonizadores, como sí ocurrió con la ablación. 
Un caso similar precisa volver a la profesora Champeil-Desplats (2010), en esta ocasión refiriéndose a los hechos ocurridos en Puno (ciudad, departamento y provincia del sureste del Perú), cuando el Gobierno decretó el carácter de patrimonio cultural para la hoja de coca y la posterior consideración de inconstitucionalidad del decreto demandado desde la Presidencia de la República. Lo anterior obedeció a dos razones: por tratarse de una competencia del órgano nacional y por la evidente incompatibilidad entre la consideración de la hoja de coca como patrimonio cultural y los tratados internacionales de lucha contra los estupefacientes. Esto terminó en un enfrentamiento cultural entre los occidentales, que eran finalmente los que le habían otorgado el uso como estupefacientes, y los andinos, para quienes la coca es apenas un elemento más de su paisaje natural, una planta proveedora de bienestar y salud, y un elemento fundamental en las ofrendas ceremoniales (Andina, 2008).

Ciertamente, la protección constitucional a los derechos a la diversidad étnica y cultural en Colombia, así como a la autonomía que gozan los pueblos indígenas están vinculadas con los tratados, convenios y declaraciones internacionales que en esta materia se han acordado (González Serrano, 2013). Sin embargo, es preciso insistir en la línea final del artículo 246 de la Constitución, que recoge la amplitud del concepto de autonomía para introducirlo definitivamente en el sendero de la gran Carta: "siempre que no sea contraria a la Constitución y a leyes de la Republica” (C. P., 1991, artículo 246, [Colom.]). En palabras de la Corte, la protección a la diversidad cultural dispuesta normativamente no implica algún tipo de distanciamiento entre la autonomía de las comunidades indígenas y la realidad jurídica nacional; por el contrario, los derechos a la autonomía y al autogobierno deben coordinarse con la ley nacional (C. C., Sentencia T-001/12). Las dos claridades definen el rumbo de la JEI en cuanto al uso de su cultura, advirtiendo que cuando exista tensión "entre 
los derechos individuales fundamentales y el reconocimiento de la diversidad étnica y cultural" (C. C., Sentencia T-001/12) corresponde al juez la decisión, teniendo en cuenta las particularidades del caso y la variabilidad de los componentes de la cultura entre una y otra etnia. Para llevar el tema de la ablación hasta un posible alcance de la autonomía indígena, se debe observar la problemática a partir de tres aspectos definitivos e innegociables: 1) la condición de etnia, 2) la condición de mujeres, y 3) la condición de niñas, donde estas últimas son sujetos de derechos con especial protección:

En principio, la competencia para resolver los conflictos relacionados con niños indígenas está en el seno de la comunidad a la que pertenecen y deben ser resueltos por sus autoridades conforme a sus usos y costumbres. En este ámbito, se debe observar el principio pro infans, que consiste en la prevalencia de los derechos de los niños sobre los derechos de los demás. Sin embargo, cuando la jurisdicción indígena o la misma comunidad viola los contenidos esenciales que forman parte de las restricciones de la jurisdicción indígena, se pueden tutelar por parte de la jurisdicción nacional los derechos de los niños indígenas, ya que estos conservan sus derechos individuales que no pueden ser negados por la colectividad. (C. C., Sentencia T-001/12)

Las Sentencias T-349/96 y SU-510/98 establecen las causas por las cuales se hace preciso limitar el desarrollo de algunas manifestaciones culturales que, de acuerdo con los principios del ius cogens, generan afectación a los derechos fundamentales. Dichas causas son las siguientes: "por el derecho a la vida (C. P., artículo 11), por las prohibiciones de la tortura (C. P., artículo 12) y la esclavitud (C. P., artículo 17) y por legalidad del procedimiento y de los delitos y de las penas (C. P., artículo 29)" (C. C., Sentencia T-001/12). Los argumentos jurídicos por los cuales la protección a estos derechos es inminente obedecen a su carácter universal, 
imprescriptible, inalienable e irrenunciable, y todos juntos hacen parte de un acuerdo intercultural que los valora en igualdad de condiciones. Los derechos no explicitados en las Sentencias T-349/96 y SU-510/98 quedan en manos del juez constitucional para que, haciendo uso de la ponderación, pueda aplicar el principio de la "maximización de la autonomía de las comunidades indígenas y la minimización de las restricciones" (C. C., Sentencia T-349/96), con el fin de evitar que el desarrollo normativo correspondiente a la JEI no sea letra muerta. En este sentido, la ablación, entendida como un procedimiento contrario al derecho a la vida y la integridad de las niñas indígenas de la etnia embera chamí, fue asumida por el Estado por poner en peligro la vida de las afectadas. En consecuencia, basándose en la prevalencia de los derechos fundamentales de las niñas y de las mujeres, las autoridades locales de Puerto Rico en el departamento de Risaralda, junto con los médicos del Hospital San Rafael, asumieron los cuidados clínicos de las tres pequeñas que ingresaron al centro hospitalario luego de que sus clítoris fueron mutilados ("Polémica por ritual embera chamí", 2007). Jurídicamente, la ablación contraviene los derechos fundamentales anotados (Morales y Daza, 2017), ya que los informes médicos evidencian las siguientes patologías: crecimiento excesivo de tejido de cicatrización, complicaciones en el parto y riesgo de muerte del recién nacido, condición quirúrgica permanente que obliga a la práctica de intervenciones para disponer el organismo a la vida sexual y al parto, riesgo de muerte para la madre por causas como hemorragia, síncope neurogénico provocado por el dolor y altos niveles de infección en el aparato reproductor; asimismo, dificultad para sanar las heridas, formación de abscesos, relaciones sexuales dolorosas, predisposición a enfermedades de transmisión sanguínea como el VIH/SIDA y la hepatitis, periodos menstruales con 
acentuado dolor, infecciones e incontinencia urinaria (Marrugo Núñez, 2014).

Por todo lo expuesto, es probable que la intervención del Estado provoque desconfianza a la hora de verificar el cumplimiento y respeto por el desarrollo de la JEI, como lo establece la Constitución. Sin embargo, dadas las condiciones de extremo riesgo en que se encontraban las niñas de la etnia embera chamí, el derecho a la autonomía por razones culturales se vio rendido ante la prevalencia de los derechos fundamentales:

[...] la consagración del principio de diversidad étnica y cultural [...] se encuentra en una relación de tensión con el sistema de derechos fundamentales consagrado en la Constitución, toda vez que, mientras el primero persigue la protección y aceptación de cosmovisiones y parámetros valorativos diversos e, incluso, contrarios a los postulados de una ética universal de mínimos, el segundo se funda en normas transculturales y universales que permitirían la convivencia pacífica entre las naciones. (Sentencia SU-510/98)

Sin embargo, el profesor Juan Carlos Abreu y Abreu menciona que los miembros de las comunidades indígenas, reconocidos a sí mismos como tales, precisan su adaptación a los principios jurídicos de los derechos humanos, apostando a la comprensión y adhesión de su cultura a estas normas, para regular la convivencia entre las culturas que se encuentran. Además, advierte que no se trata de subestimar la percepción del mundo que tienen las comunidades, sino de "compartir imperativos de regulación necesarios para el fortalecimiento del Estado" (Abreu y Abreu, 2017, p. 73).

\section{Conclusiones}

Los Estados liberales reconocidos a sí mismos como multiculturales y pluriétnicos dieron un gran paso al identificarse en su diversidad, 
condición que por muchos años fue aclamada por quienes se percibían en sus diferencias étnicas, religiosas, de género, políticas, ideológicas, etc.

Por motivos culturales, la diversidad - un amplísimo concepto que por su condición inconmensurable escapa al completo arbitraje social- obligó a los Estados a acudir a estrategias formales de validación, a partir de la interpretación normativa y jurisprudencial del orden nacional e internacional, lo cual terminó colocando la cultura a los pies de la interpretación y reinterpretación, proveniente de comunidades externas, con visiones adaptadas a la percepción social mayoritaria.

Este artículo advierte que la práctica de la ablación cumple con tres elementos de la cultura: la práctica generalizada (entre los miembros de una comunidad como la embera chamí, no ocurre lo mismo para otras comunidades), la transmisión por generaciones y la implicación de un significado para la comunidad, independiente de que implique un deterioro a la condición de mujer. Frente al caso, los Estados liberales como Colombia han tenido que acudir a instancias como la Corte Constitucional para que emitan claridad en aspectos concretos relacionados con ciertas prácticas tradicionales de algunas comunidades, como el cepo, los nacimientos múltiples (gemelos y mellizos), o el uso ancestral de la coca. Sin embargo, al llegar a la ablación, se abre un verdadero debate relacionado con la falta de precisión en algunos aspectos que la misma Constitución Política llama a proteger y preservar a partir de lo que considera cultura.

Por otra parte, es importante considerar que la interpretación de lo que es y lo que no es cultura, a la luz del derecho nacional e internacional, termina siendo definido por un ejercicio de interpretación sociológica de la norma, puesto que, al final, las tensiones culturales son dirimidas por la sociedad mayoritaria, desde su propia consideración de lo normal y legal. En el caso de la etnia embera chamí, el Estado intervino porque su 
prioridad es garantizar los derechos fundamentales de sus gobernados, en este caso, los derechos a la vida y a la sexualidad de las mujeres, independientemente de las creencias que como comunidad tengan. El particular caso que aborda este trabajo admite que no es posible preservar la identidad cultural a expensas de los derechos fundamentales.

También debe señalarse que los cientos de años de aculturación a los que estuvieron sometidas estas comunidades no permiten hablar con certeza de la existencia de culturas completamente originales. Esto rescata la necesidad de traer la cultura considerada como ancestral a la indetenible evolución del mundo contemporáneo, en lo que pasa a denominarse pluriculturalismo, que admite el rompimiento de guetos culturales para acercar a los miembros de las diferentes comunidades a una sociedad que admite sus particularidades, al tiempo que prioriza la existencia de los derechos humanos como medida de su propio desarrollo.

\section{Referencias}

Abreu y Abreu, J. C. (2017). Reflexiones para una crítica a los derechos humanos, desde una perspectiva multicultural. Revista Académica de la Facultad de Derecho de la Universidad de La Salle, 14(28), 51-78. http://repositorio.lasalle.mx/handle/lasalle/670

Bastos, S. y Brett, R. L. (comps.). (2010). El movimiento maya en la década después de la paz (1997-2007). F\&G editores.

Bastos, S. y Cumes, A. (coords.). (2007). Mayanización y vida cotidiana: análisis específicos. Facultad Latinoamericana de Ciencias Sociales. https://biblio.flacsoandes.edu.ec/libros/digital/50673.pdf

Beech, H. (5 de diciembre de 2020). El drama de las mujeres birmanas que son exhibidas como atracciones turísticas. New York Times.

https://www.nytimes.com/es/2020/12/05/espanol/mundo/tailandia-kayanturismo.html

Via Inveniendi Et Iudicandi

e-ISSN: 1909-0528 | DOI: https://doi.org/10.15332/19090528

Vol. 16 N.o 1 | enero-junio del 2021 
Bossen, L. y Gates, H. (2017). Bound feet, young hands: tracking the demise of footbinding in village China. Stanford University Press.

Cayzac, H. (2001). Guatemala, proyecto inconcluso: la multiculturalidad, un paso hacia la democracia. Facultad Latinoamericana de Ciencias Sociales.

Chaparro, L (2020). La violencia contra las mujeres cometida por parte de exparejas en Colombia: cuna cuestión familiar? En C. Barraza (ed.), Debates y desafios para los derechos humanos en Colombia (pp. 177-203). Ediciones USTA.

Constitución Política de Colombia [C. P.]. 4 de julio de 1991 (Colom.).

Corte Constitucional de Colombia [C. C.], 14 de diciembre de 1992, M. P.: E. Cifuentes Muñoz, Sentencia T-605 de 1992.

https://www.corteconstitucional.gov.co/relatoria/1992/T-605-92.htm

Corte Constitucional de Colombia [C. C.], 13 de septiembre de 1993, M. P.: E. Cifuentes Muñoz, Sentencia T-38o de 1993.

https://www.corteconstitucional.gov.co/relatoria/1993/T-380-93.htm

Corte Constitucional de Colombia [C. C.], 8 de agosto de 1996, M. P.: C. Gaviria Díaz, Sentencia T-349 de 1996.

https://www.corteconstitucional.gov.co/relatoria/1996/T-349-96.htm

Corte Constitucional de Colombia [C. C.], 15 de octubre de 1997, M. P.: C. Gaviria Díaz, Sentencia T-523 de 1997.

https://www.corteconstitucional.gov.co/relatoria/1997/T-523-97.htm

Corte Constitucional de Colombia [C. C.], 18 de septiembre de 1998, M. P.: E. Cifuentes Muñoz. Sentencia SU-510 de 1998.

https://www.corteconstitucional.gov.co/relatoria/1998/su510-98.htm

Corte Constitucional de Colombia [C. C.], 25 de enero del 200o, M. P.: F. Morón Díaz, Sentencia T-030 de 2000.

https://www.corteconstitucional.gov.co/relatoria/2000/t-030-00.htm

Corte Constitucional de Colombia [C. C.], 30 de agosto de 2006, M. P.: M. G. Monroy Cabra, Sentencia C-742 de 2006, https://www.corteconstitucional.gov.co/relatoria/2006/C-742-06.htm

Via Inveniendi Et Iudicandi

e-ISSN: 1909-0528 | DOI: https://doi.org/10.15332/19090528

Vol. 16 N. ${ }^{\circ} 1$ | enero-junio del 2021 
Corte Constitucional de Colombia [C. C.], 23 de noviembre de 2011, M. P.: J. I. Pretelt Chaljub, Sentencia C-882 de 2011. https://www.corteconstitucional.gov.co/relatoria/2011/C-882-11.htm

Corte Constitucional de Colombia [C. C.], 11 de enero de 2012, M. P.: J. C. Henao, Sentencia T-001/12.

https://www.corteconstitucional.gov.co/RELATORIA/2012/T-001-12.htm

Champeil-Desplats, V. (2010). El derecho a la cultura como derecho fundamental. Revista Electrónica Iberoamericana, 4(1), 92-116. https://www.urjc.es/images/ceib/revista electronica/vol 42010 1/REIB 041 o Veronique.pdf

Contreras Peláez, F. J. (2001). Derechos colectivos, libertad individual y mitología comunitarista en Will Kymlicka. En F. J. Ansuátegui Roig (ed.), Una discusión sobre los derechos colectivos (pp. 135-156). Instituto de Derechos Humanos "Bartolomé de las Casas"; Dykinson.

Cortés, S. (2016). Derechos humanos en las políticas de paz y posconflicto en Colombia. Revista Via Inveniendi et Iudicandi, 11(1), 129-145. https://doi.org/10.15332/s1909-0528.2016.0001.03

Gallo, A. (1988). Identidad y valor cultural (Vol. III). Universidad Rafael Landívar.

Giménez Montiel, G. (2016). Estudios sobre la cultura y las identidades sociales. Instituto Tecnológico y de Estudios Superiores de Occidente. https://books.google.com.co/books?id=fCZwDQAAQBAJ\&printsec=frontcover\&hl $=\mathrm{es} \# \mathrm{v}=$ onepage $\& \mathrm{q} \& \mathrm{f}=$ false

González Cortés, G. L. (2020) ¿Perpetúan las normas la discriminación hacia las mujeres? De la necesidad del análisis de la producción legal en Colombia. Via Inveniendi Et Iudicandi, 15(1), 119-160. https://doi.org/10.15332/19090528/5744

González Serrano, A. (2013). Obligaciones de los Estados parte de la Convención Americana. Revista Saber, Ciencia y Libertad, 8(2), 45-56. https://doi.org/10.18041/2382-3240/saber.2013v8n2.1903

Hernán Fuentes declara patrimonio cultural de Puno a hoja de coca. (2 de enero de 2008). ANDINA. https://andina.pe/agencia/noticia-hernan-fuentes-declarapatrimonio-cultural-puno-a-hoja-coca-155367.aspx

Via Inveniendi Et Iudicandi

e-ISSN: 1909-0528 | DOI: https://doi.org/10.15332/19090528

Vol. 16 N.o 1 | enero-junio del 2021 
Kuhn, T. (2007). La estructura de las revoluciones científicas. Fondo de la Cultura Económica.

Kymlicka, W. (1996). Ciudadanía multicultural: una teoría liberal de los derechos de las minorías. Paidós.

Lima Soto, R. E. (1995). Aproximación a la cosmovisión maya. Universidad Rafael Landívar, Instituto de investigaciones Económicas y Sociales.

López-Austin, A. (1989). Hombre-Dios: religión y política en el mundo Náhuatl. Universidad Nacional Autónoma de México. www.historicas.unam.mx/publicaciones/publicadigital/libros/hombre/dios.html

Ludeña Silverio, L. (8 de febrero de 2018). Unaf reúne a especialistas internacionales para abordar la mutilación genital femenina. AmecoPress.

https://amecopress.net/Unaf-reune-a-especialistas-internacionales-para-abordarla-Mutilacion-Genital-Femenina

Macías Reyes, R. (2011). El trabajo sociocultural comunitario. Fundamentos epistemológicos, metodológicos y prácticos para su realización. Editorial Académica Universitaria. http://edacunob.ult.edu.cu/bitstream/123456789/33/1/El\%20trabajo\%2osociocul tural\%20comunitario.\%20Fundamentos\%20epistemol\%C3\%B3gicos\%2C\%20meto dol\%C3\%B3gicos\%20y\%20pr\%C3\%A1cticos\%2opara\%20su\%2orealizaci\%C3\%B3 n.pdf

Marrugo Núñez, N. (2014). Multiculturalismo, derechos humanos y derechos de los indígenas en Colombia. Práctica de la ablación en las niñas de la tribu embera Chamí. Justicia Juris, 1O(1), 32-42. https://doi.org/10.15665/rj.v10i1.297

Morales, S. y Daza, S. M. (2017). El deber de información al paciente el consentimiento informado y el tratamiento ambulatorio en España. Novum Jus, 1O(2), 11-34. https://doi.org/10.14718/NovumJus.2016.10.2.1

Moraña, M. (2014). Bourdieu en la periferia: capital simbólico y campo cultural en América Latina. Cuarto Propio.

https://books.google.com.co/books?id=kgHhCQAAQBAJ\&printsec=frontcover\&d $\mathrm{q}=$ campo+cultural+bourdeau\&hl=es\&sa $=\mathrm{X} \& v e d=0$ ahUKEwiTvfO3ju3dAhWRu1M $\underline{\text { KHQDmBXUQ6AEIKTAA\#v=onepage\&q =campo\%20cultural\%2obourdeau\&f=fal }}$ $\underline{\mathrm{se}}$

Via Inveniendi Et Iudicandi

e-ISSN: 1909-0528 | DOI: https://doi.org/10.15332/19090528

Vol. 16 N.o 1 | enero-junio del 2021 
Moreno Pestaña, J. L. y Vásquez García, F. (eds.). (2006). Bourdieu y la filosofía. Montesinos.

Organización Mundial de la Salud. (3 de febrero de 2020). Mutilación genital femenina. https://www.who.int/es/news-room/fact-sheets/detail/female-genital-mutilation

Ortega, L., Cárdenas, C., Blanco, C., Ducuara, S. y Valencia, D. (2020). Reflexiones jurídicas sobre el derecho a la felicidad y el bien común constitucional. Ediciones USTA. https://repository.usta.edu.co/handle/11634/31220?show=full

Ortiz Quiroga, J. A. (2013). La identidad cultural de los pueblos indígenas en el marco de la protección de los derechos humanos y los procesos de democratización en Colombia. Revista Derecho del Estado, 30, 217-249. https://revistas.uexternado.edu.co/index.php/derest/article/view/3.524

Polémica por ritual embera chamí. Mutilación a bebés indígenas. (23 de marzo de 2007). Periódico Hoy, p. 5.

Ramírez, M. y Mazariegos, L. M. (1993). Tradición y modernidad: lecturas sobre la cultura Maya actual. Universidad Rafael Landívar.

Rodríguez, E. (2016). El pasaje del Estado y el derecho a la postmodernidad. Revista Via Inveniendi et Iudicandi, 11(2) 11-37. https://doi.org/10.15332/s1909$\underline{0528.2016 .0002 .01}$

Salazar, H. (4 de diciembre de 2010). Etnia indígena prohíbe la ablación femenina. $B B C$ News. https://www.bbc.com/mundo/noticias/2010/12/101203 colombia indigenas em bera ablacion wbm

Taylor, C. (1996). Fuentes del yo: la construcción de la identidad moderna (A. Lizón, trad.). Paidós.

Vásquez Castañeda, D. (1983). Festividades de Guatemala. Ministerio de Educación.

Waldron, J. (1992). Minority Cultures and the Cosmopolitan Alternative. University of Michigan Journal of Law Reform, 25(3-4), 751-793. https://repository.law.umich.edu/cgi/viewcontent.cgi?article $=1722 \&$ context $=$ mjlr

Via Inveniendi Et Iudicandi

e-ISSN: 1909-0528 | DOI: https://doi.org/10.15332/19090528

Vol. 16 N. ${ }^{\circ} 1$ | enero-junio del 2021 
Walsh, D. (30 de abril de 2020). In a victory for women in Sudan, female genital mutilation is outlawed. The New York Times.

https://www.nytimes.com/2020/04/30/world/africa/sudan-outlaws-femalegenital-mutilation-.html?smid=tw-share

Wilson, R. (1993). Resurgimiento Maya en Guatemala: (experiencias Q'eqchi'es). Centro de Investigación de Centro América.

Via Inveniendi Et Iudicandi

e-ISSN: 1909-0528 | DOI: https://doi.org/10.15332/19090528

Vol. 16 N.o 1 | enero-junio del 2021 